\title{
NEUTRAL GAS CONTRIBUTIONS TO \\ [S II] GALACTIC BACKGROUND EMISSION
}

\author{
S. J. PETUCHOWSKI AND C. L. BENNETT \\ NASA/ Goddard Space Flight Center \\ spet@stars.gsfc.nasa.gov
}

\section{Introduction}

Elevated ratios of [S II] $/ \mathrm{H} \alpha$ relative to those typical of $\mathrm{H}$ II regions have been used to identify supernova remnants (SNRs) and also characterize the diffuse Galactic background (Reynolds 1985) and gas observed on the large scale in other galaxies (e.g., Lehnert \& Heckman 1994). We explore the implications for these regions of the fact that ionized sulfur may exist both in gas that is predominantly neutral as well as fully ionized.

\section{Co-Occurence with [O I]}

In SNRs, strong [S II] emission may arise due to the abundance of $\sim 10^{4} \mathrm{~K}$ electrons in shock regions. These electrons are sufficiently energetic to provide $1.85 \mathrm{eV}$ excitation of S II transitions at 6716 and $6731 \AA$ and excitation of [O I] in the same, predominantly neutral, regions.

While [O I] and [N II] emission is poorly correlated across samples of 10 SNRs in M31 (Blair, Kirshner, \& Chevalier 1981) and 36 SNRs in M33 (Smith et al. 1993), we find that [S II] intensity from these regions can be decomposed into terms proportional to [O I] and [N II], as shown in Figure 1. We interpret the first term as [S II] arising within a neutral component, while the ionized component gives rise to a second term containing a factor which reflects the respective dependence of N II and S II on metallicity.

\section{Excitation via Photoionization}

[S II] emission may arise, additionally, in the absence of hot electrons, and unaccompanied by [O I] emission. The mechanism for sustaining [S II] ex- 


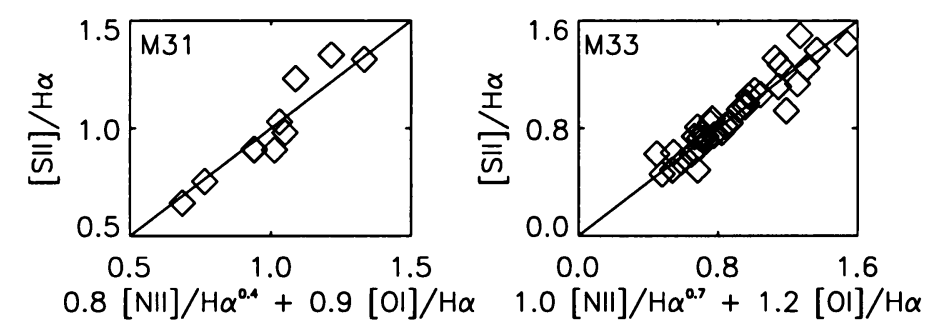

Figure 1. Fits to $[\mathrm{S} \mathrm{II}] / \mathrm{H} \alpha$ vs. $a_{i o n}([\mathrm{~N} \mathrm{II}] / \mathrm{H} \alpha)^{m}+\mathrm{a}_{\text {neut }}[\mathrm{O} \mathrm{I}] / \mathrm{H} \alpha$ for SNRs in (a) M31 (Blair et al. 1981) and (b) M33 (Smith et al. 1993).

citation in cool neutral gas is that of photoionization into electronically excited states. Of those sulfur ions photoionized in a Draine (1978) spectrum of the interstellar radiation field, approximately $25 \%$ are created in the ${ }^{2} \mathrm{D}$ level, based on partial cross sections of Chapman \& Henry (1971). Under typical conditions in a diffuse photodissociation region (PDR), the observed forbidden line intensity is governed by the rate of recombination.

The intercept of a fit to [S II] vs. $\mathrm{H} \alpha$ at zero $\mathrm{H} \alpha$ in the Galactic background fields observed by Reynolds (1985) suggests a contribution of $(0.42 \pm 0.19)$ Rayleighs to the [S II] flux from predominantly neutral gas. This intensity can arise in a thickness of photodissociated gas of unity optical depth within a range of temperatures between 37 and $120 \mathrm{~K}$ if the electron density is in the range of $1-5 \mathrm{~cm}^{-3}$ (Petuchowski \& Bennett 1995). For an electron density equal to a nominal carbon density in this boundary layer, the inferred neutral hydrogen density is $(8000 \pm 4000)$ $\left(\frac{T}{100 K}\right)^{0.593} \mathrm{~cm}^{-3}$, within the range typical of PDR interfaces of neutral clouds in the Galaxy (Hollenbach et al. 1991).

We conclude that cool neutral gas, possibly at the boundaries of fully ionized regions, constitutes a candidate medium for the origin of [S II] emission in the Galactic background.

\section{References}

Blair, W. P., Kirshner, R. P. \& Chevalier, R. A. 1981, Astrophysical Journal, 247, p. 879.

Chapman, R. D., \& Henry, R. J. 1971, Astrophysical Journal, 168, p. 169.

Draine, B. T. 1978, Astrophysical Journal Supplement Series, 36, p. 595.

Hollenbach, D. J., Takahashi, T., \& Tielens, A. G. G. M. 1991, Astrophysical Journal, 377, p. 192.

Lehnert, M. D. \& Heckman, T. M. 1994, Astrophysical Journal Letters, 426, p. L27.

Petuchowski, S. J., \& Bennett, C. L., Astrophysical Journal, in press.

Reynolds, R. J. 1985, Astrophysical Journal, 294, p. 256.

Smith, R. C., Kirshner, R. P., Blair, W. P., Long, K. S., \& Winkler, P. F. 1993, Astrophysical Journal, 407, p. 564. 